zinc in corneal tissue. It is worthy of note that in my case I used zinc sulphate for three weeks, applied every three hours, and then for another three weeks applied three times daily, and that no deposit of zinc in the tissue was apparent.

\section{CHARACTERISTICS OF THE ORGANISM.}

The Morax-Axenfeld diplobacillus, an organism which so far as known is only pathogenic for the human eye, averages about 1 micron wide and 2 microns long, the size, however, varying. When taken from the conjunctival sac the bacilli, in my experience, vary very little in width, the length being more variable as one sees both short and long ones. When grown on Loeffler's blood serum, the medium most often used for their cultivation, one sees great variations in shape and size. Taken from this medium one finds thin bacilli and thick ones, short and long ones and degeneration forms. They usually occur in pairs, end on, whether found in secretion or culture, the ends of the bacilli being slightly rounded. Frequently pairs, dividing into four, as indicated by constrictions forming in the middle of each, are met with. One may also find short chains; this is particularly so in cultures. The bacilli stain with all the ordinary dyes and are negative to Gram.

As a rule, there is not nearly so much secretion in a conjunctival sac infected with this organism as in other forms of conjunctival infection, and sometimes it is hard to obtain a mass for staining, but usually a deposit may be found on the caruncle. From such a deposit generally the bacilli are present in enormous numbers. However, variations from this occur, and sometimes, as in my Case 5, very few organisms are found. The secretion in a diplobacillus conjunctivitis consists in great part of fibrin and is poor in cells. The bacilli may lie on the cells; I have not seen bacilli within cells.

The diplobacillus can be grown only at blood temperature, and with certainty only on blood serum or serum agar or on culture media containing human body fluids. Loeffler's blood serum is a most excellent culture medium, and it is a medium which is always available, for almost every city health department supplies it for the diagnosis of diphtheria. On this medium in from 16 to 24 hours the bacilli produce a very characteristic picture, in that they liquefy the medium, forming holes in it, the bases of which are moist and without color. After a while these holes become bigger and may in due course run into one another. If the growth is allowed to continue, the culture medium may be almost completely liquefied. Of all the bacteria observed in the conjunctiva none other grows like the diplobacillus on blood serum, except the bacillus of Petit. This organism is very similar in all its characteristics to the MoraxAxenfeld bacillus, and only by cultural characteristics can it be differentiated from the latter, namely, in growing abundantly on all usual culture media and in liquefying gelatin.

[EdrToR's Note: Dr. Pusey's article was discussed with those of Drs. Shumway and Weeks. These and the discussion will be published next week.]

Rules for Resident Physicians.-J. T. Rugh, in the Jeffer sonian for May, gives the following succinct rules for the conduct of a resident physician: 1. Serve your chief by obeying his directions and those of the institution. 2. Be always ready to do your duty and as much more as time will permit. 3 . Cultivate observation and reason, and acquire as large a fund of common sense as possible. 4. Have regard to your personal appearance and let it be always neat. 5. Pay attention to details. 6. Keep the eyes and ears open and the mouth shut.

\section{TREATMENT OF LOOSENED TEETH.* \\ M. L. RHEIN, M.D., D.D.S. \\ NEW YORK CITY.}

No departure from a physiologic condition of the mouth is so readily noticed by the sufferer as a tooth which has become more or less loosened in its alveolar socket. In considering this abnormal condition of the teeth, two classes may be distinguished.

First. Those cases resulting from infection which has caused pus to spread in such a manner as to interfere with the pericemental attachment of the root to the alveolus. In such cases, the more or less speedy removal of the focus of infection will generally restore the tooth to its normal degree of solidity. Infection following a dying pulp, difficult erupting teeth, ligatures accidently left around the necks of teeth, ill-advised separating of teeth and other injudicious dental operations are the most common causes in this class, and, therefore, they can be strictly classified as localized diseases, readily cured if the cause is ascertained and removed.

Second. Those cases in which necrotic conditions prevail and the tooth bocomes gradually less firm in its socket as it loses more and more of its peridental attachment. These abnormal conditions are the gradual results of some form of malnutrition, and consequently are not so readily amenable to treatment.

\section{ETIOLOGY.}

It follows as a natural result that, in order to treat a. loosened tooth in a rational manner, the first thing is to make a correct diagnosis of the etiology of a given case. Every practitioner has his "red letter" cases among his records of very loose teeth, cured by the removal of a suppurating pulp; cases which have been brought to him after some worthy colleague has given up the case in despair; having erroneously treated as a pyorrheal pocket an ordinary alveolar abscess. Lest we find ourselves in the same class at some time or other, let us beware of hasty opinions of any case of suppuration. The etiology of some cases is more difficult to diagnose than that of others and often it will take the most careful differential diagnosis to reveal the true condition of affairs.

EXAMINATION OF THE TEETH.

The first thing in such an examination is to determine whether or not there are any gingival pockets, and if so, how far they extend toward the end of the root, and in many cases, when the pockets are very extensive, to determine how much peridental attachment is left. For this purpose, the pockets should be carefully sprayed with some sterilizing solution, and the examination is then best made by means of a very thin flat right angle burnisher. When the pocket extends completely from the gingiva all the way around the very apex of the root, it is generally safe to assume that there is no vitality left in the pulp of the tooth and it becomes the first duty of the operator to enter the pulp of such a tooth and to remove every portion thereof. When these pockets are of a very insignificant depth and often not at all perceptible as real pockets, the question as to the condition of the pulp is a more serious one. Recourse can now be had to the different means at our disposal of testing for pulp vitality by thermal and electric effects. This, however, is not always satisfactory. The condition of

* This paper, illustrated by lantern slides made from radiographs of clinical cases, was read in the Section on Stomatology of the American Medical Association, at the Fifty-seventh Annual Sesslon. June, 1906. 
occlusion of the particular tooth is an important matter for consilleration. It is not uncommon to find a pericementitis set up, followed by the loosening of a tooth which has all the strain of occlusion of the entire jaw centered on it, caused, perhaps, by some recent filling which has not been sufficiently ground away. It should be remembered that, in an acute inflammation of a loosened tooth, there is apt to be at times some protrusion of the tooth itself from the socket. This in itself will produce a stress of occlusion at this particular point, and while it has become a detrimental factor, care should be taken not to confound it with the original cause of the trouble.

THE USE OF THE ROENTGEN RAYS.

Since the advent of the Roentgen ray in practice, it has been found of great aid in diagnosing pathologic

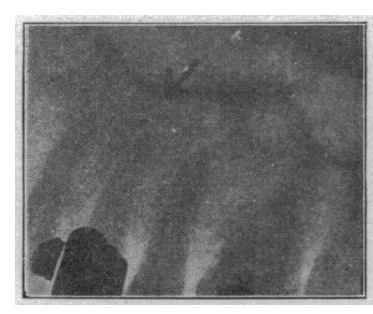

Figure 1.

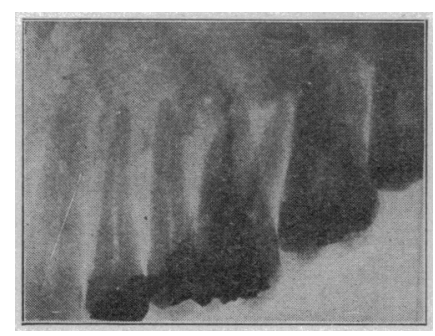

Figure 2. conditions, both in the pulp and around the roots of the teeth. Whenever there is any doubt on the subject of the vitality of the pulp, it is almost imperative at the present day to have a radiograph made of the part involved. For this purpose the film should be small enough to embrace only two or three teeth adjoining the affected one, for the purpose of obtaining a picture as free from distortion as possible. In a number of cases of loosened teeth, in which all the former methods of examination have proved unsatisfactory, a radiograph will frequently show the outlines of a well-defined abscess in the vicinitv of the apex of the root. Whenever this pericemental abscess is found it is useless to attempt to treat it without thoroughly removing the pulp contents, for it is not an uncommon thing to find vitality still remaining in the

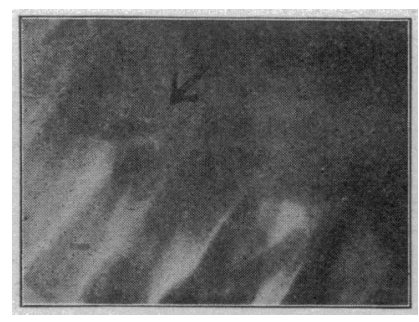

Figure 3.

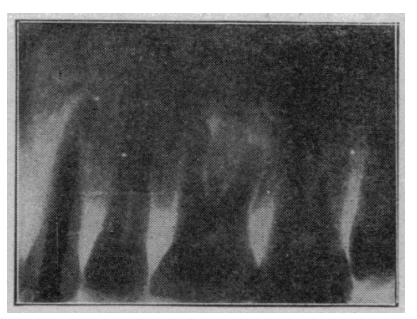

Figure 4. pulps of such teeth. The results of the examination and treatment in many such cases leads me to form the opinion that it is only a question of time before the infected zone will cut off the vitality of the pulp and all this material become added fuel to the inflammatory fires raging in this locality. If this conclusion is based on the results of correct observation, it follows as a natural conclusion that it is a wise thing to remove such a pulp aseptically before it has been reached by the infected material.

In many cases in which there is no question of the tooth having lost its vitality, it is impossible to determine from ordinary observation whether such a dead pulp has been removed by a previous operator, and, if re- moved, whether the root has been properly filled. For this purpose the radiograph is also a very valuable aid, as, under proper conditions, it will disclose the evidence of most of the generally used methods of root filling and by this means can be ascertained whether the root canal has been thoroughly filled or not. In the same manner, when suspicion exists of the penetration of the antrum by the root of a tooth, a radiograph showing the same is of value in corroborating the diagnosis.

It is not an easy matter to read a radiograph correctly. The practitioner starting to use this diagnostic field must make up his mind to study the pictures carefully at the beginning, or he will be liable to form false deductions from what he thinks he sees. This is most apparent in a picture of an upper molar, when frequently the floor of the antrum dips down between the lingual and buccal roots. The radiograph will show the

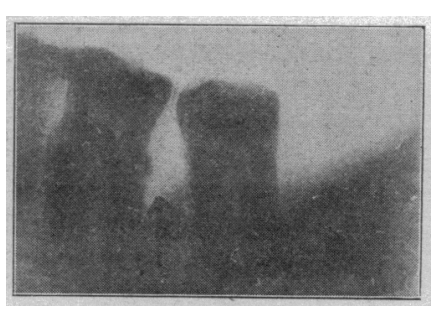

Figure 5.

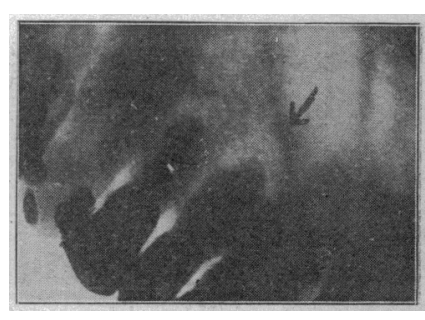

Figure 6. floor of the antrum well defined, and apparently all the roots of the tooth penetrating the same. The result will be that the beginner will suppose that all the upper molars which he examines have their roots penetrating the antrum. In the same way there are root-filling materials which will not appear in a radiograph. Later I will show a slide (Fig. 6) of an upper bicuspid, the canal of which appears not to have been filled. On entering this root canal it was found to be filled with cotton to the very end. This particular root had given no trouble for over twenty years, and during this time the tooth had never been disturbed. An examination showed a well-defined pericemental abscess and slight absorption around the end of the root, and yet this cotton was found

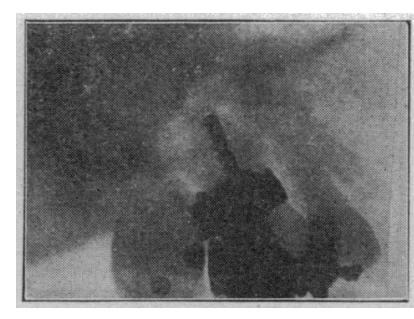

Figure 7.

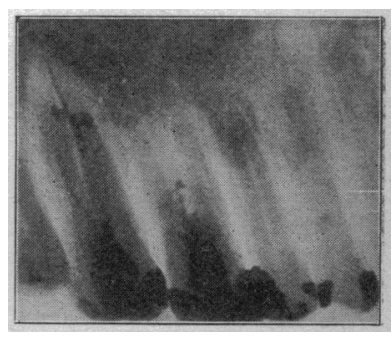

Figure 8. free from odor or taint of infection, it being, in fact, the only root filling of cotton that ever came under my observation that appeared to the eye to be in anything like a sterile condition. The patient had commenced to suffer from pain around the end of the root, due undoubtedly to the abscessed area in this locality, and this caused the taking of the radiograph which plainly showed the seat of trouble. The picture was read incorrectly, as it led to the false inference that the canal was unfilled. This case is dwelt on to some extent, because it illustrates so forcibly that the radiograph, while of great value as an aid in diagnosis, can not be absolutely depended on ; consequently, this drawback should never be lost sight of by the careful diagnostician. 
There are a large number of traumatisms coming under the head of the first class of cases which, producing a pericementitis, will be often followed by considerable loosening of the tooth. In such cases a good radiograph is of inestimable value, inasmuch as it will show up, generally speaking, an entirely healthy field, so far as the alveolar socket is concerned (Fig. 1, inflammation from imperfect gold crown). This will indicate that, with proper treatment, the tooth will return to its normal condition, and at any rate delay is indicated so far as any active operative measures are concerned.

In the second class of cases in which malnutrition under some of its manifold and devious methods is playing havoc with the nutritive supply around the alveolar sockets, the consensus of opinion at the present time
Figure 9

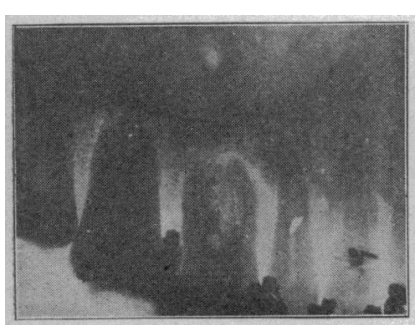

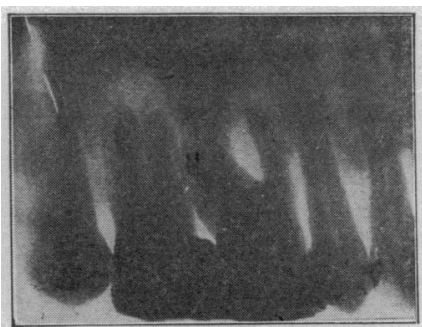

Figure 10 appears to be that in all such cases the pulp has ceased to retain its physiologic characteristics. Lack of time prevents a proper consideration of the details of this subject. It can be stated that the removal of all such pulps is a well-recognized and satisfactory form of treatment; whenever it is feasible every vestige of pulp tissue should be removed to the very end of the different roots. To this end the original radiograph will be found of great service as a guide to the operator while removing the pulp tissue.

It is best perhaps to state that in all these cases every particle of deposit and foreign matter has been carefully removed, the surfaces of the teeth have been thoroughly polished and the pockets treated both surgically and therapeutically. The patient is instructed to brush his teeth and gums carefully four times a day, and this frequent massage of the gums has a very invigorating influence on the capillary circulation. The teeth are to be retreated at the expiration of three or four weeks. In addition to this massage of the gums by the use of the tooth brush in the patient's hand, the therapeutic use of the Roentgen ray has been more or less advocated during the past two years. It has not been in use Iong enough for us to form a positive view as to its real value. There is a strong unanimity of opinion among careful observers that in many cases it has exerted a very favorable influence toward an improved nutritional condition. But its use for this purpose has been mostly of an empirical nature, and on this account it is necessary to advise great caution. No absolute definition of the means by which the circulatory condition is improved from the use of the $x$-ray has been discovered. It is a well-known fact that in all investigations the $x$-ray has shown no germicidal properties. The general belief is that the circulatory corpuscles are stimulated to a great degree. and yet this is a very indefinite statement. Furthermore, it is necessary to use the $x$-ray with great care and caution on account of the liability of inflammation arising from a too prolonged application. Its use might be safely started with the application of a strength of five amperes at a distance of about twelve inches. The length of time of the exposure can be safely started at about one and a half minutes and gradually increased according to the judgment of the operator. The high frequency current in various forms is also used for this purpose and has the one advantage that it appears to lack the dangerous qualities inherent in the Roentgen ray.

\section{OTHER METHODS OF TREATMENT.}

Frequently, treatment of this nature will suffice to cause sufficient repair to the deteriorated tissues so that the tooth will again become firm in its socket. In order to accomplish this result, it is essential to remember that every possible pus focus must be removed and one of these foci that frequently escapes the observer is a root of one of the multirooted teeth that has lost its entire attachment and is absolutely a necrotic appendage to the rest of the tooth. Such a root (Fig. 8) must invariablv be amputated and removed in order to remove from the field permanently every possible focus of infection. After such a necrosed root has been removed, the best results can only be attained by replacing it with a porcelain substitute, the technic of which I have described elsewhere ${ }^{1}$ (Fig. 7). After a trial of more than seven years, it can be safely asserted that this operation is a very valuable means for the preservation and tightening of loosened teeth. About 85 per cent. of the cases operated on have resulted favorably; considering the fact that some of the cases were experimental, the results achieved are very satisfactory.

A tooth that is loose and wobbly is only a source of discomfort and the irritating nature of such a tooth materially tends to increase the unhealthy condition of its own environment. A tooth in such a condition should either be removed, or, if saved, it must be placed in such a state that it will cease to be a source of discomfort and irritation. When the different methods of treatment previously outlined have failed, there remains the last resource of unit-

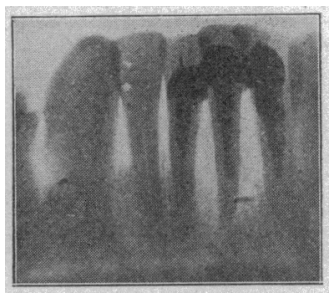

Figure 11.

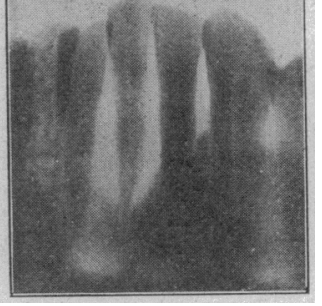

Figure 12
Figure 13.

ing loosened teeth to each other, or to teeth which are in $\cdot a$ healthy condition. This operation ${ }^{2}$ has been found invariably successful in the retention of teeth that seemed doomed to the forceps (Figs. 11 and 13).

The one thing to remember in the treatment of all the loose teeth of the second class is that after the mouth has been made comparatively comfortable and the loosened teeth firm this condition can only be maintained by careful prophylactic treatment once every month at the hands of the dentist and dental nurse.

1. Items of Interest, February, 1904, and Dental Cosmos, September, 1902

2. Dental Cosmos, March, 1888, and Id., May, 1903. 
No'te: Dr. Khein then exhibited on the screen a large number of lantern slides of radiographs taken from clinical cases. $A$ few of these are here reproduced.

Figure 1 illustrates a normal physiologic maxilla with the exception of a gold shell cap over the first bi-euspid. This tooth had been somewhat loose from the irritation produced by this badly constructed cap, and the radiograph shows distinctly that the trouble does not extend much beyond the gingiva of this tooth. The removal of the cap is sufficient to cause the tightening of the loosened tooth.

Figure 2 is a radiograph of the mouth of a man, aged 54 . It has a number of interesting features, but the main one centers around the apical portion of the roots of the second molar, which shows a well-defined abscess, although no suspicion of this existed. After this radiograph had been taken the pulp cavity was at once entered and found to be filled with a foul-smelling, putrescent material. In this mouth there were four other teeth in like condition, and the patient's general condition was very much deteriorated as a result of so much septic matter. The removal of these numerous pus foci caused a rapid and marked improvement in the general condition.

Figure 3 shows another mouth with practically the same history. Over the first bi-cuspid can be seen the outlines of a well-marked pericemental abscess. The same condition is evident in the first molar. Neither of these teeth had any fillings or defects in their crowns.

Figure 4 is a radiograph of the same mouth a week after the putrescent pulp had been removed from the first bi-cuspid, and the root had been filled. There is already evident a marked improvement in the tissue conditions around the apex of the root of the first bi-cuspid.

Figure 5 demonstrates the effects of calculary deposit on the side of the root of a molar, and one that has escaped the visual examination of the operator.

Figure 6 illustrates the case described at length of a root Eilled with cotton over twenty years, when suddenly an intense neuralgic condition started up around the end of the root, due to the abscess shown at the point of the arrow in the illustration. The artificial crown on the first bi-cuspid distinctly shows the root filling, but the fact that the root of the second bi-cuspid has been filled is not at all perceptible in the radiograph, and illustrates the point of the necessity of careful study of radiographs in order to be able to read them properly. This particular abscess was cured by the removal of the cotton root-filling and the application of electrolysis through the end of the root for a period of seven minutes with three milliamperes of current.

Figure 7 is a radiograph of an upper molar after the lingual root had been removed and replaced by a porcelain substitute. The root that is visible is the porcelain substitute.

Figures 8,9 and 10 show three different stages in the mouth of a man 55 years of age who had been a sufferer from arthritic pyorrhea alveolaris for years. There were numerous pericemental abscesses in this mouth that were located by means of the radiograph. The first and second molars here illustrated were so loose that they wobbled from side to side and could have been easily removed by the patient himself.

Figure 8 illustrates that the pulp of the second molar had been thoroughly removed and the roots filled to the very end. It also indicates that the lingual root had lost all its attach. ment and was practically a foreign body and should be removed. The first molar was found to be in a still worse condition. The previous dentist had not been able to find the canals of the buccal roots. The lingual root in this tooth is also entirely devoid of attachment and partly absorbed. The first thing accomplished in this case was reopening into the pulp cavity of the first molar and the thorough cleansing of the buccal roots and filling of the same as shown in Figure 9 . The lingual roots of both teeth were then amputated and porcelain substitutes for these made. Two iridiumized platinum staples were bent into shape; one extending from the porcelain root to the second molar across the occluding surfaces and into the pulpal cavity of the first molar and vice versa; a staple from the porcelain root of the first molar, across the occlusal surfaces into the pulpal cavity of the second molar. These staples were fastened in with amalgam, which entirely contoured the crowns of both molars, making a perfect splint, and holding the two teeth rigidly together.

Figure 10 is from a radiograph showing the complete operation of the two teeth splinted together and showing the two porcelain substitutes in place of the necrotic roots that were amputated.

Figure 11 illustrates a superior central incisor with very bad pyorrheal pockets. The tooth was very loose and could be moved in and out at random with the tongue. The wide space between this and the adjoining incisor made the case more than usually difficult. The illustration shows the condition of the parts after it had been successfully splinted to the adjoining lateral incisor.

Figure 12 is a radiograph of a pyorrheal condition surrounding the lower incisor, which, as is readily apparent, was almost ready to drop out of the mouth.

Figure 13 illustrates the method used to preserve this incisor in a firm condition, retaining all the esthetic beauty of this and the adjoining teeth. The splint here shown is only observable on the lingual side and can not be seen without the aid of a mouth mirror.

38 East Sixty-first Street.

\section{INTERSTITIAL GINGIVITIS DUE TO AUTOINTOXICATION}

\section{AS INDICATED BY THE URINE AND BLOOD PRESSORE -DIAGNOSIS.* \\ FUGENE S. TALBOT, M.S., D.D.S., M.D., LL.D. chicago.}

In a former paper, "Interstitial Gingivitis Due to Autointoxication," I discussed etiology; in my work on "Interstitial Gingivitis, or So-called Pyorrhea Alveolaris" I demonstrated the pathology; in this paper I propose to discuss the method of diagnosis in interstitial gingivitis. It has been demonstrated that this disease originates from autointoxication. It is justifiable, then, to state that interstitial gingivitis is dependent on the circulation and the vasomotor system. It is, then, a profitable subject for investigation. Before discussion of this phase of the subject, it is necessary to become familiar with the structures involved, which in themselves are unique. There are no other structures in the human body like them. I have gone into details many times in a description of these structures. I will now only discuss them in a general way. The alveolar process is a bony structure intended to hold the tecth in position; when the teeth are removed the process absorbs; therefore, it is a transitory structure. In the evolution of the head, face, jaws and teeth, the process including the jaws grows smaller, the teeth and process change their shape, making the alveolar process doubly transitory. Transitory structures are more easily involved by disease than any other. Some of the lower vertebrates shed their teeth continuously. A tooth comes into place, is used a short time, is shed and a new one takes its place. This continues throughout the life of the animal. Man has two sets. He sheds the first, after they have performed their functions. and a new set takes its place. As soon as the alveolar process has developed about them a low form of inflammation sets up absorption. I have called this osteomalacia or senile absorption. This form of absorption will continue until the teeth loosen and drop out or are extracted. It is an atavistic process. The alveolar process is an end organ. The tooth, so far as the process is concerned, is a foreign body. The arteries, capillaries and nerves pass through the bony process and stop.

- Read in the Section on Stomatology of the American Medical Association, at the Fifty-seventh Annual Session, June, 1906. 